\title{
Impact of digitalization of mammographic units on average glandular doses in the Flemish Breast Cancer Screening Program
}

\author{
An De Hauwere, Hubert Thierens \\ Ghent University, Department of Medical Physics, Proeftuinstraat 86, B-9000 Gent, Belgium \\ an. dehauwere@uGent. be
}

\begin{abstract}
The impact of digitalization on the average glandular doses in 49 mammographic units participating in the Flemish Breast Cancer Screening Program was studied. Screen-film was changed to direct digital radiography and computed radiography in 24 and 25 departments respectively. Average glandular doses were calculated before and after digitalization for different PMMAphantom thicknesses and for groups of 50 successive patients. For the transition from screen-film to computed radiography both phantom and patient dose data show a significant increase of dose with digitalization. For the transition from screen-film to direct digital radiography the evolution of the average glandular dose depends on the phantom thickness. For 20mm PMMA a significant increase in dose was found, for $45 \mathrm{~mm}$ and 70mm PMMA there was a significant decrease in dose. The median average glandular dose of the patient dosimetry showed a smaller but significant decrease.
\end{abstract}

Keywords: mammography, screening, breast cancer, digitalization, average glandular dose, screen-film, computed radiography, direct digital radiography

\section{Introduction}

After a typetesting procedure [1-2] the first digital mammographic units were accepted within the Flemish Breast Cancer Screening Program in 2007. It is often claimed by manufacturers and in the media that "digitalization" in mammography leads to a dose reduction. However over the past 5 years, medical physicists in Flanders encountered that for individual mammographic centers the dose is sometimes higher after digitalization than before. Since asymptomatic woman between age 50 and 69 are systematically screened for breast cancer every two years in Flanders, it is necessary to assure the balance between benefit and the risk of the screening program. Therefore it is important to check the impact of the changes in average glandular dose caused by digitalization of the screening units on the radiation-induced breast cancer risk of the Flemish Breast Cancer Screening Program. 


\section{$2 \quad$ Method}

The impact of digitalization on the average glandular doses was studied in 49 mammographic units participating in the Flemish Breast Cancer Screening Program. Screen-film (SF) was changed to direct digital radiography (DR) and computed radiography (CR) in 24 and 25 departments respectively.

For all centers data regarding the yearly and half yearly physical-technical quality assurance test are available, in accordance with the European Guidelines [3-4]. On one hand average glandular doses (AGDs) before and after digitalization could be calculated for PMMA-phantom thicknesses between $20 \mathrm{~mm}$ and $70 \mathrm{~mm}$ applying the method of Dance et al. [5] based on automatic exposure control data, combined with output and half value layer measurements.

On the other hand a real patient dose study was performed in all centers after digitalization. Average glandular doses were calculated for a group of at least 50 successive patients. A large fraction of the centers conducted also a patient dose study prior to digitalization. Periodic patient dose studies are required by Belgian legislation [6]. A first registration period ended in the beginning of 2010. At that time some mammographic centers were already working digital, others were still working conventional. For each center that digitalized later on, a second patient dose study was requested.

For each patient dose study, the median of the average glandular doses was plotted against the average glandular dose corresponding with the $45 \mathrm{~mm}$ PMMA-phantom at the time. These data could be represented satisfactory by a linear fit $(\mathrm{y}=1.066 \mathrm{x} \pm$ $0.235, \mathrm{r}^{2}=0.738$, Fig. 1). For centers who did not perform patient dosimetry of a patient population prior to digitalization, the linear fit was used to deduce the median of the average glandular doses for a group of patients from the phantom dose data obtained prior to digitalization.

AGDs before and after digitalization were compared for each mammographic unit individually and significance of difference was evaluated by Wilcoxon signed rank test $(\mathrm{p}<0.05)$. Mean AGDs were calculated over the different units.

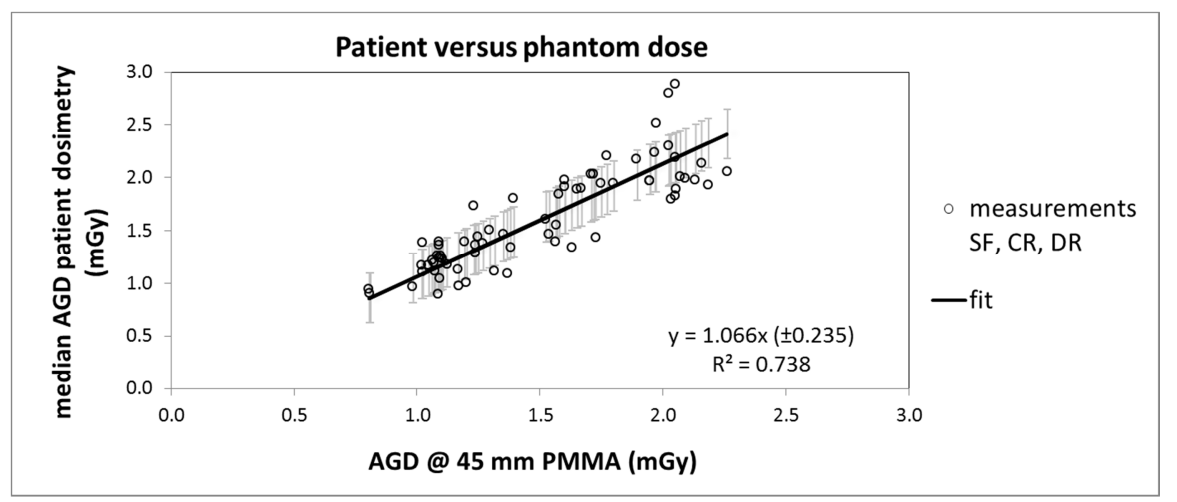

Fig. 1. Correlation between the median of the AGDs of a group of 50 patients and the AGD of a 45mm PMMA phantom 


\section{$3 \quad$ Results}

Before digitalization there was a large variation in average glandular dose as a function of phantom-thickness between the 49 mammography units (Fig. 2). The dose differences were mainly due to the brand and type of the screen-film combination. For larger compressed breast thicknesses the observed dose differences are also linked to the brand and type of the corresponding x-ray units. Older x-ray units operate at $\mathrm{Mo} / \mathrm{Mo}$ for all thicknesses. However the automatic exposure control of newer x-ray units changes at larger compressed breast thicknesses automatically to other target/filter combinations and/or higher kilovoltages, which reduces the AGD in such a way that the image quality remains acceptable.

For all CR-systems phantom-doses are quite high and there is a smaller variation in doses between the different brands of CR-cassettes (Fig. 3). This because the automatic exposure controls had to be calibrated close to the acceptable dose limit stated in the European Quality Control Protocol in order to reach acceptable image quality for small details.

For DR-systems phantom-doses are generally lower than for CR-systems (Fig. 4) and comparable to the lower dose SF-systems. Only one DR-system operated at a dose level lower than the lowest SF-system, this was a mammography unit with a photon counting detector instead of an amorphous silicon or amorphous selenium detector.

These trends were also seen in the patient dosimetry, though differences in individual breast composition and in the executed compression force have an influence on the correlation between phantom en patient dosimetry.

For the transition from SF to CR (25 screening units) phantom AGD increased significantly for 20mm, 45mm and 70mm PMMA in nearly all mammographic units (Fig. 5, Fig. 6, Fig. 7, Table 1). The median AGD of a dose study of 50 successive patients was also significantly higher after digitalization from SF to CR (Fig. 8, Table 1).

For the transition from SF to DR (24 screening units) the evolution of the AGD depends on the phantom thickness. For 20mm PMMA a significant increase in dose was found (Fig. 5, Table 2). For $45 \mathrm{~mm}$ and 70mm PMMA there was a significant decrease in dose (Fig. 6, Fig. 7, Table 2). The median AGD of a dose study of 50 successive patients showed a smaller but significant decrease after digitalization from SF to DR (Fig. 8, Table 2). 


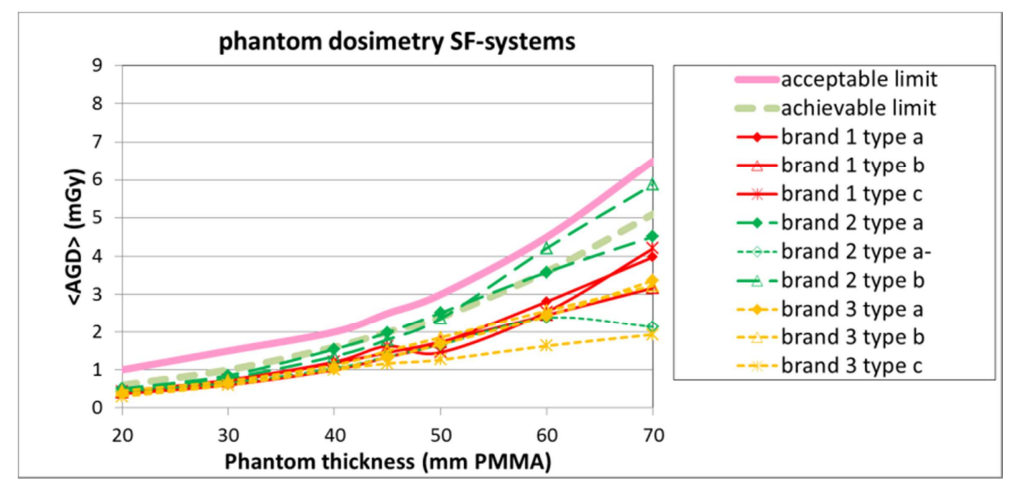

Fig. 2. Screen-film phantom dosimetry: AGD versus phantom thickness averaged over different brands and different screen-film combination types

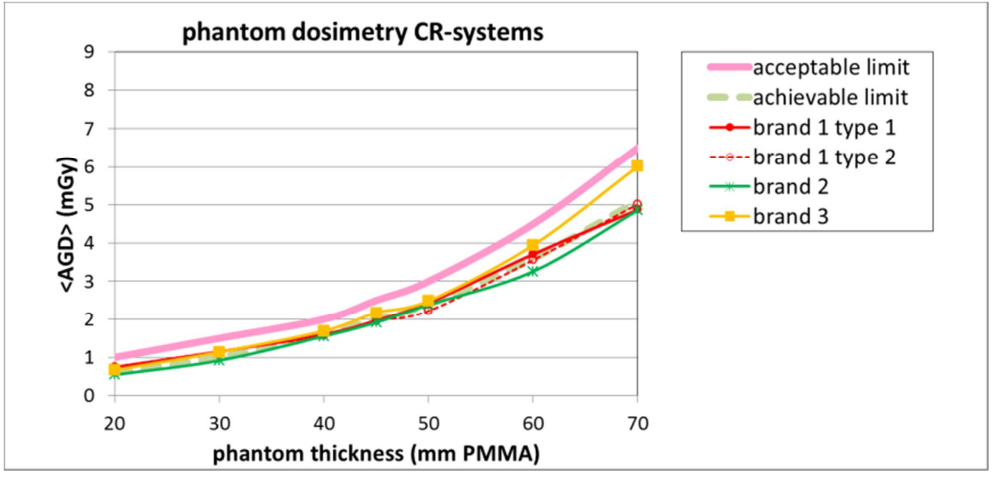

Fig. 3. Computed radiography phantom dosimetry: AGD versus phantom thickness averaged over different brands and different imaging plate types

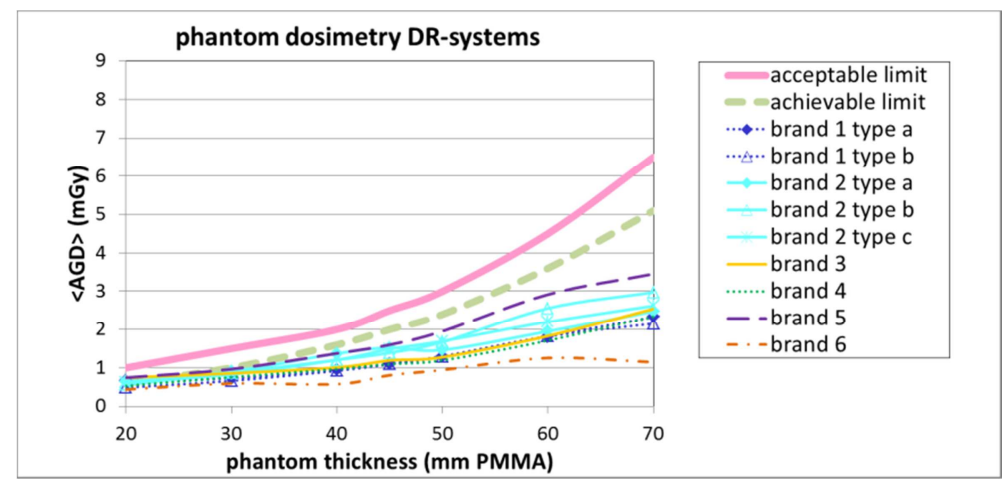

Fig. 4. Direct digital radiography phantom dosimetry: AGD versus phantom thickness averaged over different brand $\mathrm{s}$ and different models of mammography equipment 


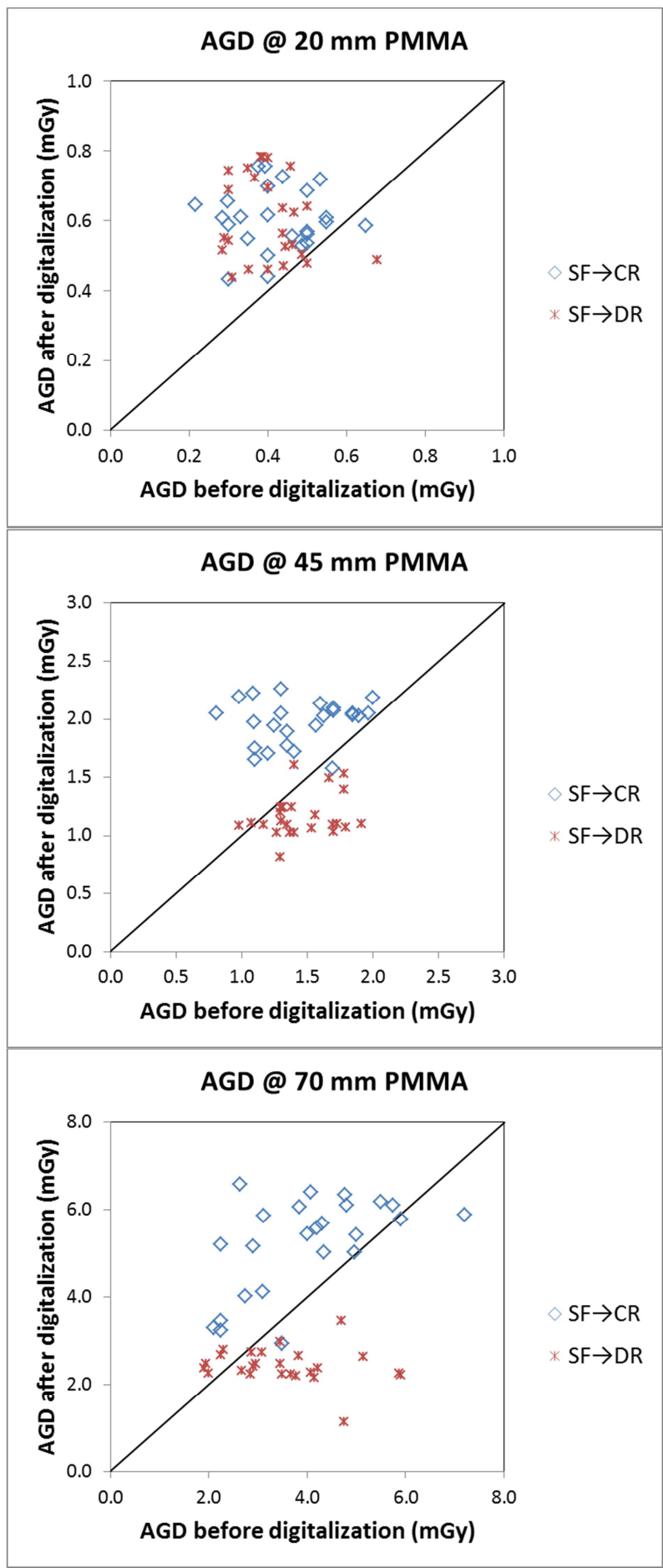

Fig. 5. Phantom dosimetry at 20mm PMMA: AGD before and after digitalization in the mammographic units of the study

Fig. 6. Phantom dosimetry at 45mm PMMA: AGD before and after digitalization

Fig. 7. Phantom dosimetry at 70mm PMMA: AGD before and after digitalization 


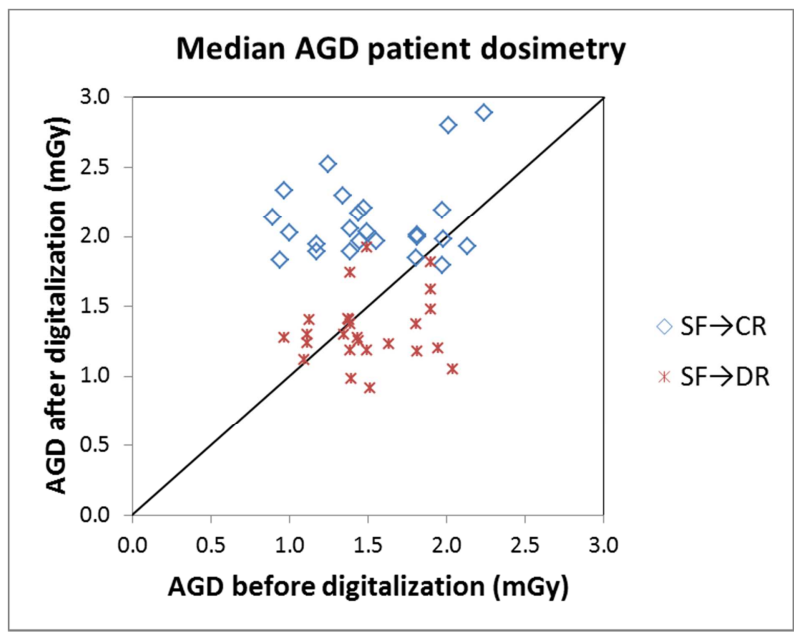

Fig. 8. Median AGD of a patient dose study of 50 successive patients before and after digitalization

Table 1. Statistical analysis of the transition from SF to CR

\begin{tabular}{|l|c|c|c|c|}
\cline { 2 - 5 } \multicolumn{1}{c|}{} & $\begin{array}{c}\mathrm{SF} \\
\text { mean }\end{array}$ & $\begin{array}{c}\mathrm{CR} \\
\text { mean }\end{array}$ & $\begin{array}{c}\text { normalized dose } \\
\text { difference }\end{array}$ & $\mathrm{p}^{*}$ \\
\hline AGD @20 mm PMMA (mGy) & 0.42 & 0.60 & $43 \%$ & 0.000 \\
\hline AGD @45 mm PMMA (mGy) & 1.45 & 1.97 & $36 \%$ & 0.000 \\
\hline AGD @70 mm PMMA (mGy) & 3.98 & 5.21 & $31 \%$ & 0.000 \\
\hline median AGD patient dosimetry (mGy) & 1.53 & 2.11 & $38 \%$ & 0.000 \\
\hline
\end{tabular}

* Wilcoxon Signed Ranks Test

Table 2. Statistical analysis of the transition from SF to DR

\begin{tabular}{|l|c|c|c|c|}
\cline { 2 - 5 } \multicolumn{1}{c|}{} & $\begin{array}{c}\mathrm{SF} \\
\text { mean }\end{array}$ & $\begin{array}{c}\text { DR } \\
\text { mean }\end{array}$ & $\begin{array}{c}\text { normalized dose } \\
\text { difference }\end{array}$ & $\mathrm{p}^{*}$ \\
\hline AGD @20 mm PMMA (mGy) & 0.41 & 0.61 & $49 \%$ & 0.000 \\
\hline AGD @45 mm PMMA (mGy) & 1.45 & 1.16 & $-20 \%$ & 0.000 \\
\hline AGD @70 mm PMMA (mGy) & 3.53 & 2.43 & $-31 \%$ & 0.001 \\
\hline median AGD patient dosimetry (mGy) & 1.50 & 1.32 & $-12 \%$ & 0.032 \\
\hline
\end{tabular}

* Wilcoxon Signed Ranks Test

\section{Discussion}

It is a misconception that digitalization in mammography always leads to a dose reduction. Our results demonstrate that the transition from screen-film mammography to computed radiography on average in fact leads to a $38 \%$ higher AGD for an average patient. This higher dose is necessary to reach an acceptable image quality level for small details. In contrast a reduction of 12\% in AGD was achieved on average for an average patient with a transition from screen-film mammography to direct digital radiography. We believe a stronger dose reduction for the transition from SF to DR can be accomplished by optimizing the automatic exposure control of the DR sys- 
tems. This because for 20mm PMMA phantom doses are higher for DR than for SF. From our yearly and half yearly test results, we know that image quality in terms of contrast-to-noise ratio is much higher at 20mm PMMA than at larger thicknesses. As the European Quality Control Protocol [4] demands a constant contrast-to-noise ratio for all thicknesses, there is a potential of dose reduction for DR-systems at smaller thicknesses. Care must be taken that the overall contrast-to-noise level stays high enough to see small low-contrast details even in dense and thick breasts.

Whether and to what extent the patient dose decreases or increases in an individual mammographic unit after digitalization therefore largely depends on the choice for $\mathrm{CR}$ or for DR and is also determined by the brand and type of the formerly used screen-film combination and $\mathrm{x}$-ray unit.

An increase of $38 \%$ in average glandular dose for the transition from SF to CR could change the detection over induction ratio (DIR) of the Flemish breast cancer program from 40 to 30 provided that the cancer detection rate stays the same. Therefore we plan to investigate for the same 49 mammography units, the impact of digitalization on the breast cancer screening performance parameters such as recall rate, cancer detection rate, fraction of invasive cancers, fraction of invasive cancers smaller than $1 \mathrm{~cm}$, fraction of ductal carcinoma in situ (DCIS) and the positive predictive value. This in order to calculate the DIR for digital mammography screening in Flanders and to ensure the overall quality of the digitalized Flemish breast screening program.

\section{Conclusion}

In present study it was shown that transition from screen-film to digital mammography will not necessarily result in a dose reduction. In fact the use of computed radiography leads to an important increase in average glandular dose $(38 \%)$. On the other hand direct digital mammography contributed to a small but significant decrease in average glandular dose (12\%). Direct digital mammography can be further optimized for smaller compressed breast thicknesses without compromising image quality.

From patient dose point of view, direct digital mammography has to be preferred over computed radiography in digitalization of mammography screening. A close collaboration between radiographers and medical physicists is indicated.

Manufacturers of direct digital mammography systems are advised to investigate how the automatic exposure control can be further optimized for smaller thicknesses: care should be taken that clinical as well as physical image quality parameters meet all requirements in lowering the dose setting.

Results obtained in present study are clearly in favor of direct digital radiography in digitalization of the screening program.

\section{References}

1. Thierens H., Bosmans H., Buls N., Bacher K., De Hauwere A., Jacobs J., Clerinx P.: Typetesting of physical characteristics of digital mammography systems: first experiences within the Flemish Breast Cancer Screening Programme. JBR-BTR, 90, 159-162 (2007) 
2. Thierens, H., Bosmans, H., Buls, N., De Hauwere, A., Bacher, K., Jacobs, J., Clerinx P.: Typetesting of physical characteristics of digital mammography systems for screening within the Flemish breast cancer screening programme. EJR, 70, 539-548 (2009)

3. Van Engen, R., van Woudenberg, S., Bosmans, H., Young, K., Thijssen, M.: The European protocol for the quality control of the physical and technical aspects of mammography screening: Screen-film mammography. In: Perry, N., Broeders, M., de Wolf, C., Törnberg, S., Holland, R., von Karsa, L., Puthaar, E. (eds.) European guidelines for quality assurance in breast cancer screening and diagnosis. Fourth Edition. Part 2a, pp. 61-104. Office for Official Publications of the European Communities, Luxembourg (2006)

4. Van Engen, R., Young, K., Bosmans, H., Thijssen, M.: The European protocol for the quality control of the physical and technical aspects of mammography screening: Digital mammography. In: Perry, N., Broeders, M., de Wolf, C., Törnberg, S., Holland, R., von Karsa, L., Puthaar, E. (eds.) European guidelines for quality assurance in breast cancer screening and diagnosis. Fourth Edition. Part 2b, pp. 105-165. Office for Official Publications of the European Communities, Luxembourg (2006)

5. Dance, D.R., Skinner, C.L., Young, K.C., Beckett, J.R., Kotre, C.J.: Additional factors for the estimation of mean glandular dose using the UK mammography protocol. Phys. Med. Biol., 45, 3225-3240 (2000)

6. Royal Decree of 20 July 2001 laying down the general rules and regulation for the protection of the general public, of the workers and the environment against the danger arising from ionising radiation. Chapter VI: Medical Applications of ionising radiation. Belgian Law Gazette, 171 (245), 28982-28996 (2001) 